\title{
Disrupting Industries with Blockchain: The Industry, Venture Capital Funding, and Regional Distribution of Blockchain Ventures
}

\author{
Maximilian Friedlmaier \\ Technical University of Munich \\ Blockchain Research Group \\ maximilian.friedlmaier@tum.de
}

\author{
Andranik Tumasjan \\ Technical University of Munich \\ Blockchain Research Group \\ andranik.tumasjan@tum.de
}

\author{
Isabell M. Welpe \\ Technical University of Munich \\ Blockchain Research Group \\ welpe@tum.de
}

\begin{abstract}
The blockchain (i.e., a decentralized and encrypted digital ledger) has the potential to disrupt many traditional business models. This study investigates the emerging blockchain business-application landscape by analyzing its industry, venture capital funding, and regional distribution. By matching four venture databases on blockchain-based startups we create a unique database to analyze the technology from a diffusion of innovation theoretical perspective. First, our results show that blockchain startups are present across all industry segments and are most prominently represented in the Finance \& Insurance and Information \& Communication industries. A finegrained analysis of financial services yields increasing novel applications in existing service offerings. Second, we find that mainly Finance \& Insurance and Information \& Communication industries are funded by venture capital, but that blockchain startups are present across all industries. Third, our regional distribution analysis of the emerging ventures identifies two leading geographical blockchain clusters (i.e., the US and UK).
\end{abstract}

\section{Introduction}

The blockchain (i.e., a decentralized and encrypted digital ledger) was recently acknowledged as one of the top 10 emerging technologies by the World Economic Forum 2016 [6]. Prior to this notable acknowledgement, The Economist published several articles in its print edition about "the trust machine" $[43,42]$ and therefore introduced this remarkable new technology to a broader public audience. Prior to these developments, information science and business practice already started to explore the vast potential of blockchain technology with numerous proofs of concepts apart from its origin - developing cryptocurrencies (e.g., Bitcoin [34]). The decentralized and tamperproof ledger technology is expected to have far more use cases than digital currencies with bitcoin as its most prominent application [40].

Whereas the blockchain's cross-industry potential may be considered huge [33], it is unclear how blockchain startups are operating across industries and product/service categories. Moreover, there does not exist a comprehensive overview of neither the actual distribution of venture capital (VC) funding across the industries nor the location of blockchain startups. However, for understanding the advancement of blockchain technology and its current state, investigating these underresearched aspects of blockchain technology operations is crucial. In this vein, it is important to differentiate between theoretical blockchain applications and their business implementation to gauge the disruption potential of extant business models by blockchain technology. By mapping the existing blockchain activities and analyzing how it is used across industries and regions, we can gain an understanding of the disruptive potential of blockchain technology apart from purely conceptual considerations and can evaluate the technology from a diffusion of innovation theoretical perspective [cf. 38].

Hence, the aim of this study in answering these research questions is threefold. First, this study investigates the state of the blockchain landscape by examining the distribution of blockchain-based startups across industries, and, in particular, the occurrence of product/service categories in financial services. Taking into account the distribution of the identified startups across industries, we analyze how entrepreneurs evaluate possible applications across industries and service categories. Second, we match the distribution of startups with the allocation of venture capital investment by analyzing how funding is allocated across industries up until today. Third, we analyze to what extent blockchain technology is a global phenomenon and identify startup clusters by 
investigating where blockchain activity is concentrated. We do so by merging four different startup databases ("Blockchain Ecosystem Database", "VentureRadar", "Coindesk" and "Crunchbase"). Thus, we build the most comprehensive overview of the current blockchain landscape to provide a profound deep dive into blockchain business applications.

The paper is organized as follows. In section two, we discuss the theoretical framework for the current blockchain landscape. Subsequently, we present the empirical setting with its data set and describe the results in section four. We are concluding the paper with a discussion of the findings and their theoretical and practical implications.

\section{Theoretical Framework}

In the following sections, we set the theoretical background by introducing basic principles of blockchain technology and perceptions of it being a trust machine. In Section 2, we give an overview on essential principles of disruptive innovation, whereas Section 3 defines the attributes of the diffusion of innovation theory. The section concludes with a theoretical overview of research on business model applications for blockchain technology.

\subsection{The trust machine}

Digital currencies have been suffering two evident problems until the introduction of bitcoin. So far, central institutions (i.e., a central bank for currencies) have provided trust to the system with time-stamped transactions and, thus, assured the integrity of the system. The absence of a central source of trust raises the issue of system integrity with two important issues. First, there needs to be a guarantee that nobody can spend the same money twice. This issue is known as the double-spending problem [24]. On the other hand, the decentralized system needs to operate within an environment where participants cannot be sure of the trustworthiness of other participants. This issue takes the name the Byzantine's generals problem [25]. Nakamoto's bitcoin protocol solves this problem with its consensus protocol. It provides trust even without a central intermediary and works on a peer-to-peer network; hence, it is fully decentralized. The protocol builds on three basic pillars in order to provide neutral trust within the system: decentralization, consensus, and cryptography.

Decentralization means that the database is distributed with participants in the system. In the case of the bitcoin database, everyone has the possibility to possess a full copy (i.e., act as a node [52]). This has in a big advantage: Hackers would need to get through not just one central institution or central server in order to manipulate the whole system, but various copies of that. Hackers are facing a peer-to-peer network where there is no server, centralized service, or hierarchy in the network [1]. This makes the database less vulnerable to attacks. But it also enables nodes that have gone offline for some time to update their database and reintegrate into the system.

Nevertheless, it might be possible that more versions of the database exist. Participants have to agree on the correct source of truth, with some kind of voting rights, in order to agree on one specific blockchain [29]. This process of agreeing on one correct source of truth is a consensus and it is the second pillar of blockchain technology. Nakamoto's [34] consensus builds upon the Proof of Work concept introduced by Back [2]. It relies on computational power and its inherent costs: Once a mathematical problem is solved by brute-forcing it-hence using computational power and energy - the node (or miner) has the right to add a block to the blockchain. Participating nodes subsequently can rapidly verify the correctness and add the block onto their database. The longest chain within the system represents the actual single source of truth within the bitcoin blockchain system [34].

However, for understanding the mechanisms of blockchain technology, the third pillar, "cryptography," is crucial. In the bitcoin blockchain and the Proof of Work, some cryptographic technologies are necessary for digital signatures and data integrity-i.e., public/private keys and secure hash algorithms [19]. First, by applying the concept of public and private keys, the bitcoin blockchain assures the authenticity of participants sending transactions within the system. Public keys, first introduced by Diffie and Hellman [16], are used to correctly identify accounts (i.e., bitcoin accounts) and private keys to authenticate the user or possessor of bitcoins, respectively. This concept also finds use in encrypted website communications such as HTTPS [4]. The private key in the bitcoin protocol is used to sign the instruction to transfer bitcoins from the owner's account to another account; therefore, it gives an assurance that the transaction originally came from the initial bitcoin owner [4]. While the public key itself refers to publicly available data of a bitcoin account, it helps to verify the initiation of transactions (Figure 1).

This implies that the bitcoin is not a coin but rather a chain of digital signatures [34]. Nakamoto's protocol uses private keys to sign transactions that contain the new owner's public key and the previous transaction history of the electronic coin (i.e., bitcoin). Hence, 
participants in the system can assess the transaction history of every single electronic coin.

In addition to public and private keys, the SHA256 cryptography is applied within the bitcoin blockchain [34]. This algorithm generates hash values that have certain characteristics, which, in combination with all three pillars, make the bitcoin protocol unique. SHA-256 [19] generates a one-way unique hash value [13]. A one-way hash signifies that the input data always derives a specific hash value, but it is economically unfeasible to reconvert the hash value to its original data input. Once the data input becomes (just slightly) altered, a completely uncorrelated new hash value results from SHA-256 encryption. These properties ensure that SHA-256 and its resulting hash values find use in various applications such as digital signatures and data integrity [13]. Especially data integrity is one of blockchain technology's key features.

For every transaction, a unique hash value is calculated (see Figure 1). Numerous transactions are then bundled in a Merkle tree [32], i.e. resulting in one unique aggregated hash value for all transactions combined. Together with the hash of the previous block, the time stamp, and a nonce, they form the block itself. The nonce represents the mathematical problem of the Proof of Work concept developed by Nakamoto. Overall, the chaining of blocks ensures than no one can manipulate data without first needing to redo the work to find solutions to the mathematical problems.

Hence, the three pillars of blockchain technology — namely decentralization, consensus, and cryptography-build the foundation for the tamperproof ledger that is the focus of attention these days. To describe the different pillars, the bitcoin blockchain, as the first and largest blockchain, presents an explanatory example. Various different approaches, however, exist for specific parts of decentralization, consensus, and/or cryptography in other blockchain solutions. Nonetheless, the abovedescribed features of blockchain technology generally result in the perception that blockchains serve as trust machines [43].

\subsection{Diffusion of innovation theory}

Rogers [38] defines five attributes for the diffusion of innovation: relative advantage, compatibility, complexibility, trialability, observability. The higher the degree of these attributes, the higher the possibility of a high rate of adoption. The only exception represents the degree of complexibility, where a lower complexibility is supporting adoption while higher degree hinders a faster adoption of an innovation. A more detailed definition of Rogers'[38, p. 252ff.] attributes is presented below:

\begin{tabular}{ll}
\hline $\begin{array}{l}\text { Relative } \\
\text { advantage }\end{array}$ & $\begin{array}{l}\text { The degree to which an } \\
\text { innovation is perceived as } \\
\text { being better than the idea it } \\
\text { supersedes }\end{array}$ \\
\hline Compatibility & $\begin{array}{l}\text { The degree to which an } \\
\text { innovation is perceived as } \\
\text { consistent with the existing } \\
\text { values, past experiences, and } \\
\text { needs of potential adopters }\end{array}$ \\
\hline Complexibility & $\begin{array}{l}\text { The degree to which an } \\
\text { innovation is perceived as } \\
\text { relatively difficult to } \\
\text { understand and use }\end{array}$ \\
\hline Trialability & $\begin{array}{l}\text { The degree to which an } \\
\text { innovation may be } \\
\text { experimented with on a limited } \\
\text { basis }\end{array}$ \\
\hline Observability & $\begin{array}{l}\text { The degree to which the results } \\
\text { of an innovation are visible to } \\
\text { others }\end{array}$ \\
\hline
\end{tabular}

\subsection{Revolution or evolution: Essentials of disruptive innovations}

The digital transformation of business models makes it necessary for companies to elaborate a strategy to manage (radical) changes in value creations [22, 46]. Matt, et al. [28, p. 340] formulate four different dimensions for such digital transformation strategies: use of technologies, changes in value creation, structural changes, and financial aspects. Blockchain technology, however, affects the first two of their dimensions as the technology can be utilized to bypass middlemen in the process of value creations and reduce frictions within systems. It therefore has the potential to be disruptive.

In its initial context, disruption describes a development where a smaller company-initially focused on the lower and least profitable end of the market-rises toward a challenging competitor of incumbents [7] in the highly profitable customer segments. Over the past 20 years, the academic discussion about a general definition of disruption was and is still ongoing [53, 8, 39, 27, 20]. While most literature follows Christensen's proposition of disruption [53], his theory does not allow clearly differentiating between low-performing technologies and initially inferior technology $[41,51]$, nor does it offer a solution to the measurability of its degree of disruptiveness [21]. It is possible to determine only in retrospect whether a technology really was disruptive. 
In contrast to disruptive innovation, sustainable innovation represents improvements that are either incremental or major but that still enhance the service offering for the most profitable customers [9]. This is normally reflected in product and process innovations but also in innovating entire business models [5], where business models can be defined as "the rationale how an organization creates, delivers and captures value" [36]. Furthermore, technology shifts are not only a problem of technology innovation, but also have a close relation to the core of business models [44]. If technological innovation develops a new technology, then this affects business models as well. It could totally change how companies, or in this case incumbents, create, deliver, and capture value [44]. If managers of incumbents overlook the rise of a new technology with lower costs, higher performance, and/or better fit to customer needs, then they face a huge risk of disruption and eventually becoming insignificant. Blockchain technology could represent one of such.

\subsection{Business model and services across industries}

The three-pillar basis of the blockchain protocol emerged in 2008 [34]. The nature of blockchain protocols relies on three main principles: decentralization, cryptography, and consensus. The combination of these principles allows creating a tamperproof database (also referred to as a ledger [18]) that had its first use case with cryptocurrencies, such as the bitcoin. Since the blockchain protocol is applicable to a variety of transaction ledgers beyond cryptocurrency applications, it is now being considered for applications in other business segments within the financial services industry [37].

Blockchain technology serves as a ledger for fast transactions [33], providing trust [15] within a system of unknown users. Even though some dispute the costefficiency of the bitcoin protocol [35, 33], the World Economic Forum recently emphasized the potential in the banking industry with its report on the future of the financial infrastructure [50]. The UniCredit bank published its view on the impact of blockchain technology in the banking industry [45], stating its impact on payments, know-your-customer processes, trade finance, and post-trading [as well: 17]. The SWIFT Institute also expects "substantial reductions in both cost and risk" within certain business areas of financial services [26]. In capital markets, blockchains could affect, among other business models, clearing houses, exchanges, brokers, or remittances [30]. A similar result comes from Deutsche Bank Research, which sees the highest potential in real-time money transfer, cryptocurrencies, and settlement [14]. Insurance, another part of financial services, also holds potential for blockchain technology applications. In a recent study, McKinsey identifies the following applications: among others, automation by smart contracts, easier fraud detection, and reduction of administrative costs [31]. Hence, in financial services, there are many possibilities for applying blockchain technology. This could allow entrepreneurs to harness this potential.

Apart from financial applications, a distributed ledger has potential for use in other industries as well. In their book The Blockchain Revolution, D. Tapscott and A. Tapscott evaluate a large number of further applications beyond the financial services industry, creating a "Blockchain Utopia" by predicting ${ }^{1}$ a pure peer-to-peer economy and the return of data ownership to users [40]. They identify application potential especially in public services, another prominent industry with regard to ledgers. The UK Government Office for Science published a recent report on the potential of blockchain technology in governmental services [49]. The report identifies use cases in protecting critical infrastructure, departments for work and pensions, as well as possibilities in the improvement of international aid systems, and potential within the area of taxation.

When building a peer-to-peer economy, however, concepts such as the prosumer and the retail customer (for instance, as extant in the energy sector) become important. In these cases, the self-supplying consumer produces more energy than necessary and sells the surplus. With blockchain technology, this consumer could reach the retail energy consumer and get a retail energy price rather than a wholesale price from the big intermediary. A recent study by the Verbraucherzentrale Nordrhein-Westfalen (NRW) and PwC [48] assesses the potential of blockchain technology within the energy sector. A decentral controlled transaction and energy delivery system could be one possible use case. In addition, smart contracts could find application in further contract and document management [48].

Overall, the extensive potential of blockchain applications across industries and product or service offerings is evident. Our research assesses this potential and its use based on a comprehensive data set of blockchain startups to map out the current blockchain landscape from different perspectives. On ${ }^{1}$ Or, as they would personally state, the achieved rather than
predicted future. 
the one hand, this is from an "entrepreneur's perspective," when analyzing the evaluation of entrepreneurs of possible blockchain applications by taking into account the distribution of startups across industries. On the other hand, it is from a "venture capitalist's perspective," by analyzing the amount of venture capital funding allocated across industries up until today.

\section{Data set and methodology}

We use four data sets from different databases to derive a comprehensive overview of existing blockchain startups. We created and analyzed the final data set as of June 15, 2016. It contains 1,140 startups that use blockchain technology as part of their business models. In order to provide a holistic view of the current blockchain landscape, we use four different databases (for detailed information, see Appendix A). First, the open-source Blockchain Ecosystem Database [3] helped generate an initial list of blockchain startups. The Blockchain Ecosystem Database [3] classifies the different ecosystems into their sector and product or service category. At the time of the data collection, it contained 923 ecosystems. This initial list was extended with three additional databases: VentureRadar [47], CoinDesk [10], and Crunchbase [12]. From VentureRadar [47], we identified 336 startups with the search query "blockchain." The news and insights company CoinDesk [10] provided a further selection of bitcoin and blockchain startups. Using their database helped to identify another 137 startups. Finally, we searched the venture capital database Crunchbase [12] for further startups. By searching the short description section of the database with the queries "blockchain" and "bitcoin," we were able to merge another set of 267 startups with the existing data set. In total, we derived a list of 1,663 startups. After correcting for double listings and updated company names, all startups were analyzed and categorized in industries and product or service classes (when it has not been classified in the databases already). Existing classifications were revised and adjusted where a more intuitive denomination was necessary. We removed from the dataset any ecosystems classified as cryptocurrencies or digital currencies, as they are not pure blockchain startups but rather different blockchain protocols with little market capitalization and, by nature of blockchain protocols, are not startups.

Understanding the current expectations of the market potential of blockchain technology requires deriving a financial distribution within blockchain- related investments. Thus, the next step was to merge the list of 1,140 categorized startups with venture capital funding data extracted from the Crunchbase database [11].

\section{Results}

This section first gives an overview from the entrepreneur's perspective, and subsequently provides the venture capitalist's perspective. The regional distribution of the blockchain landscape completes the three-dimensional analysis approach outlined above.

\subsection{The blockchain landscape from an entrepreneur's perspective}

Figure 1 presents the entrepreneur's view on blockchain technology applications. The 1,140 startups show the distribution of blockchain startups across industry sectors in which entrepreneurs harness the potential for blockchain application.

With a $42.4 \%$ share, the Finance \& Insurance sector represents the largest share of blockchain applications. The second-largest group of startups operates in the Information \& Communication industry with a $36.5 \%$ share, emphasizing the origin of the technology in the area of data and information.

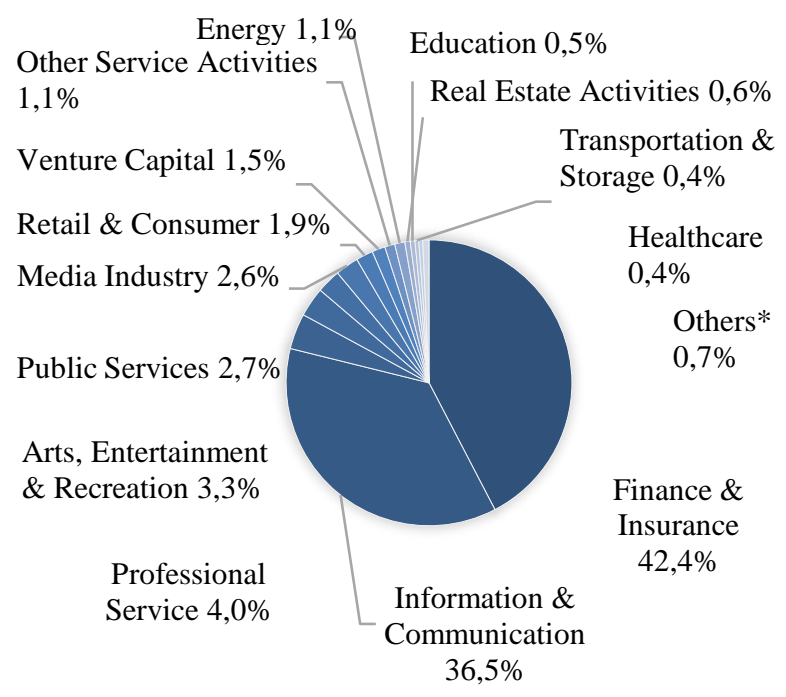

Figure 1. Percentage of startups operating in each industry sector.

Professional services (e.g., business consulting, lawyers, coaching, audit) take the third-largest share of $4 \%$. As blockchain technology is a complex new technology, the provision of professional services is necessary for companies that have little to no 
involvement in the technology so far, in order to assess potential implications for their business model(s). The media industry takes $2.6 \%$ of the total share, with startups covering expert opinions as well as legal, regulatory, and general developments within the blockchain industry.

Not only do traditional blockchain industries appear in the data set but so do industries that have not been associated with blockchain technology right from the beginning. The Arts, Entertainment \& Recreation sector represents $3.3 \%$ of blockchain startups. Next come Public Services (2.7\%), Retail \& Consumer (1.9\%), Energy (1.1\%), and Healthcare $(0.4 \%)$.

\subsection{Product or service categories in Finance \& Insurance}

In total, we identified 23 different product and service offerings in the data set for the Finance \& Insurance sector (Figure 2). One can clearly see the influence of bitcoin and cryptocurrencies - the origin of blockchain technology - when looking at the leading service category of Figure 3, Financial Exchanges \& Trading. With 181 of 483 startups in the Finance and Insurance sector, Exchanges and Trading services still constitute the center of blockchain technology

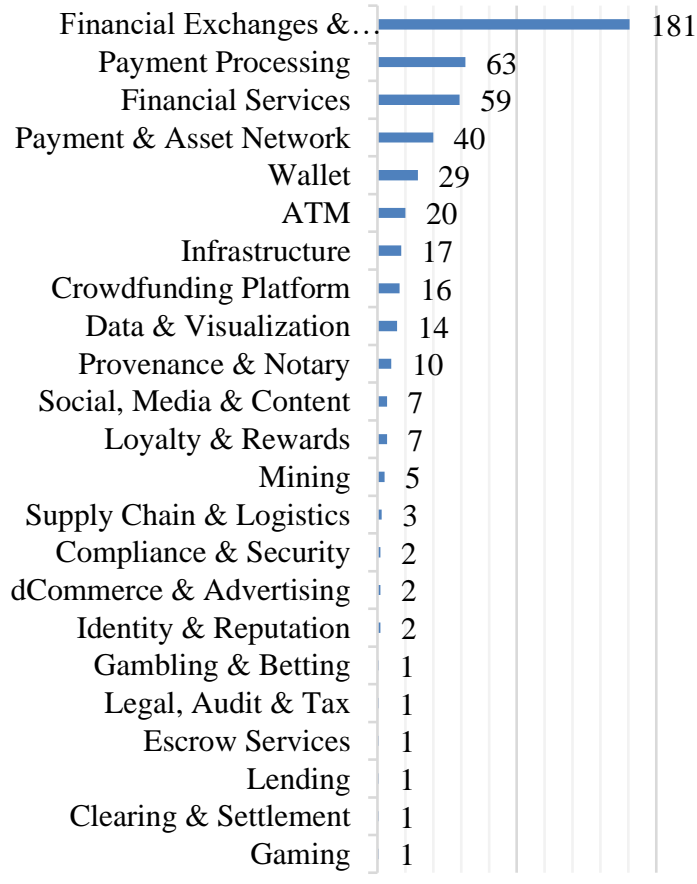

Figure 2. Number of startups by products and services in Finance \& Insurance sector
On the other hand, Payment Processing (63 startups) as well as Payment \& Asset Networks (40 startups), together with general Financial Services (59 startups), make up nearly as big a portion of business models as Financial Exchanges \& Trading itself. Crowdfunding Platforms with 16 startups, represent another application of blockchain technology within the Finance and Insurance sector. In the lower third of the figure, the representation of different business models is very fragmented. It reduces to single-figure representations.

\subsection{The blockchain landscape from a venture capitalist's perspective}

As a comparison to the entrepreneur's perspective, we add a venture capitalist's perspective to the assessment of the blockchain landscape. Table 1 shows the distribution of venture capital funding across sectors. Investments in startups of the data set sums up to USD 1,547 million. The biggest industries in terms of having received funding are Finance \& Insurance as well as Information \& Communication. Both industries together received nearly USD 1,500 million of venture capital investment, representing approximately $97 \%$ of the overall funds. While the Finance \& Insurance industry received USD 805.6 million in investments from risk-seeking investors, Information \& Communication received USD 694.33 million. When comparing the average funding of startups within the data set, both industries show the same average funding per startup of USD 1.67 million

The table shows a huge difference between the two sectors and the remaining industries from the data set. While Finance \& Insurance, and Information \& Communication are the only two sectors with more than USD 500 million as investment, eight out of 19 industries have not received any funding at all. It is notable that the Energy sector, with 13 startups in the data set, does not show any investment in their blockchain applications at all. The same holds for e.g. Healthcare (five startups), Education (six startups), and Transportation \& Storage (five startups).

Nonetheless, similar to the entrepreneur's perspective, Professional Services startups are the third-largest industry in terms of venture capital investment. Until mid-2016, this sector received USD 20.11 million in investment, with, on average, USD 0.44 million invested per startup. This positions the Professional Services sector as the only one in the double-digit space.

The Arts, Entertainment \& Recreation sector ranks fourth in terms of absolute startup numbers and remains on the same position in terms of absolute venture capital funding. But its average investment of 
USD 0.26 million per startup is smaller than the comparable sectors: Retail \& Consumer with USD 0.35 million investment per startup and Venture Capital with USD 0.37 million funding per startup. Although positioned seventh in terms of numbers of startups, Retail \& Consumer received USD 7.62 million; therefore, it ranks fourth by venture capital funds as one industry with bigger potential of blockchain technology.

Table 1.Venture capital funding of blockchain startups in total.

\begin{tabular}{|c|c|c|c|}
\hline Sector & $\begin{array}{l}\text { VC } \\
\text { funding, } \\
\text { in million } \\
\text { USD }\end{array}$ & $\begin{array}{l}\text { Number of } \\
\text { startups }\end{array}$ & $\begin{array}{l}\text { Avg. VC } \\
\text { funding } \\
\text { in million } \\
\text { USD }\end{array}$ \\
\hline $\begin{array}{l}\text { Agriculture, Forestry \& } \\
\text { Fishing }\end{array}$ & - & 2 & - \\
\hline $\begin{array}{l}\text { Arts, Entertainment \& } \\
\text { Recreation }\end{array}$ & 9.80 & 38 & 0.26 \\
\hline Education & - & 6 & - \\
\hline Finance \& Insurance & 805.60 & 483 & 1.67 \\
\hline Food \& Beverages & - & 1 & - \\
\hline Healthcare & - & 5 & - \\
\hline $\begin{array}{l}\text { Information \& } \\
\text { Communication }\end{array}$ & 694.33 & 416 & 1.67 \\
\hline Other Service Activities & 0.18 & 13 & 0.01 \\
\hline Professional Service & 20.11 & 46 & 0.44 \\
\hline Public Services & 1.11 & 31 & 0.04 \\
\hline Real Estate Activities & 1.30 & 7 & 0.19 \\
\hline Telecommunications & - & 1 & - \\
\hline $\begin{array}{l}\text { Transportation \& } \\
\text { Storage }\end{array}$ & - & 5 & - \\
\hline Venture Capital & 6.30 & 17 & 0.37 \\
\hline Media Industry & 0.55 & 30 & 0.02 \\
\hline Energy & - & 13 & - \\
\hline Tourism Industry & 0.18 & 3 & 0.06 \\
\hline Aviation \& Space & - & 1 & - \\
\hline Retail \& Consumer & 7.62 & 22 & 0.35 \\
\hline Grand Total & 1,547.08 & 1,140 & 1.36 \\
\hline
\end{tabular}

\subsection{Regional distribution of blockchain landscape}

As evident from Figure 3, the distribution of blockchain startups across the globe nearly covers the biggest regions. Figure 3 shows that the US hosts the largest group of startups - a total of 365 . The UK ranks second with regard to the assembly of startups in the blockchain landscape, with 127 startups and startup headquarters. Nevertheless, startups in the US outnumber the second largest cluster in UK by three times. Table 2 shows the absolute distribution of startups across the globe as well as the percentage of VC funding. The 29 countries represent $90.79 \%$ of the total startup distribution of 68 different countries in the data set. North America is the most penetrated region hosting 397 startups, including the US with 365 startups and Canada with 32.

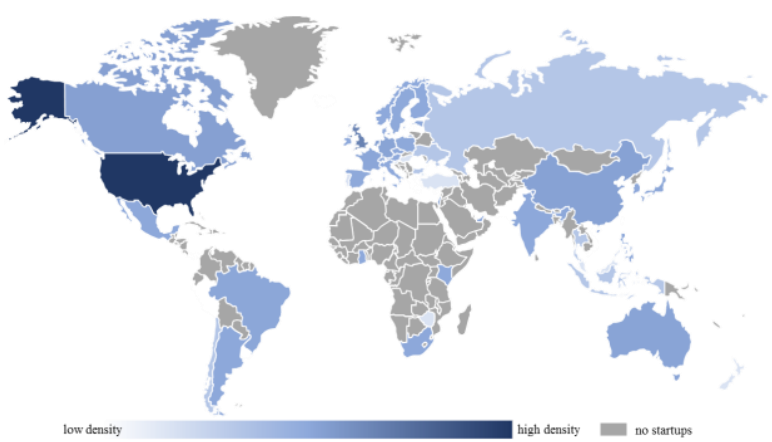

Figure 3. Color-coded world map of blockchain startup density.

Europe is close behind North America, with 300 startups. The European countries in Table 2 with the highest count of blockchain startups are led by the UK with 127 startups, and followed by the Netherlands (28), Germany (22), Switzerland (18), France (14), Spain, Poland, Sweden, and Estonia (seven each).

Table 2. Venture capital funding of blockchain startups in total.

\begin{tabular}{|c|c|c|c|c|}
\hline Country & $\#$ & $\begin{array}{l}\% \text { of Country } \\
\text { funding }\end{array}$ & \# & $\begin{array}{r}\% \text { of } \\
\text { funding }\end{array}$ \\
\hline United & 365 & $50.1 \%$ Japan & 13 & $3,0 \%$ \\
\hline $\begin{array}{l}\text { States } \\
\text { No HQ* }\end{array}$ & 221 & 10.7\% Hong Kong & 13 & $0,1 \%$ \\
\hline UK & 127 & $2.5 \%$ Mexico & 9 & $0,2 \%$ \\
\hline Canada & 32 & 7.1\% Argentina & 8 & $0,0 \%$ \\
\hline Netherlands & 28 & $4.3 \%$ South Africa & 8 & $0,0 \%$ \\
\hline China & 27 & $1.2 \%$ India & 8 & $0,1 \%$ \\
\hline Singapore & 27 & $\begin{array}{l}1.7 \% \text { Republic of } \\
\text { Korea }\end{array}$ & 8 & $0,8 \%$ \\
\hline Germany & 22 & $0.6 \%$ Spain & 7 & $0,0 \%$ \\
\hline Australia & 20 & $0.2 \%$ Poland & 7 & $0,0 \%$ \\
\hline Israel & 19 & $1.8 \%$ Sweden & 7 & $2,1 \%$ \\
\hline Switzerland & 18 & $0.2 \%$ Philippines & 7 & $0,0 \%$ \\
\hline France & 14 & $0.3 \%$ Estonia & 7 & $0,1 \%$ \\
\hline Brazil & 13 & $0.0 \% \ldots$ & $\ldots$ & $\ldots$ \\
\hline & \multicolumn{2}{|r|}{ Total startups } & $\overline{140}$ & \\
\hline $\begin{array}{l}\text { N. America } \\
\text { (N.A.) }\end{array}$ & 397 & $57.2 \%$ Asia & 140 & $9.9 \%$ \\
\hline $\begin{array}{l}\text { Americas } \\
\text { w/o N.A. }\end{array}$ & 42 & $0.7 \%$ Oceania & 22 & $0.3 \%$ \\
\hline Europe & 300 & $21.2 \%$ Africa & 18 & $0.1 \%$ \\
\hline
\end{tabular}

*Set if companies do not specify their headquarters' location or if they operate in a decentralized manner.

Asia follows as the third-biggest region in the data sample with 140 startups. Funding, however, is mainly 
clustered in the U.S. with $50.1 \%$ of total VC funding worldwide. Overall North America is the leading region in terms of funding, followed by Europe.

Of the startups, $19.39 \%$ either explicitly specified operating in a fully decentralized manner or did not publish any information about the location of their headquarters' destination. Making it into the top three leading categories from a geographical perspective and together with the US and UK counting more than one hundred blockchain ventures. The distribution of blockchain startups is spread all around the world and is increasing steadily, with some primary clusters in North America (US) and Europe (UK).

\subsection{Diffusion of innovation for blockchain technology}

With the distributions of blockchain ventures across industries, VC funding and regions the different perceived attributes of innovation according to Rogers [38]. Table 3 shows the deduction of the four attributes of Rogers' diffusion of innovation theory.

Table 3. Blockchain technology's perceived attributes of innovation [cf. 38].

\begin{tabular}{ll}
\hline $\begin{array}{l}\text { Perceived attributes } \\
\text { of innovation }\end{array}$ & $\begin{array}{l}\text { Deduction for blockchain } \\
\text { technology }\end{array}$ \\
\hline Relative advantage & $\begin{array}{l}\text { Blockchain technology offers the } \\
\text { possibility for (data related) } \\
\text { products and/or services that are } \\
\text { cheaper, faster and more secure } \\
\text { than existing technologies. }\end{array}$ \\
\hline Compatibility & $\begin{array}{l}\text { With blockchain technology } \\
\text { current market frictions can be } \\
\text { overcome by creating peer-to-peer } \\
\text { networks in many senses. Results } \\
\text { show that mainly current business } \\
\text { models and industries are prone to } \\
\text { this innovation. }\end{array}$ \\
\hline Blockchain technology relies on \\
three pillars: decentralization, \\
consensus, and cryptography. This \\
unique combination a priori makes \\
it rather difficult to understand.-------
\end{tabular}

\section{Discussion}

This quantitative part of the study examines how blockchain technology is disrupting industries from three different angles: First, we analyze the distribution of blockchain startups by determining the allocation of startups across industry sectors. By diving into the Finance \& Insurance and Information \& Communication sectors, we evaluate different applications of the new technology within existing business models. Subsequently, we consider the allocation of venture capital investment by distinguishing the current funding of blockchain applications across industries. Both analyses combined show the advancements of the disruption caused by blockchain technology across industries. The regional allocation of blockchain startups delivers an additional view of the current landscape, with two rising clusters. Examining the industry distribution of startups, the "home turf" of blockchain technologyapplied as a distributed transaction ledger for cryptocurrencies - explains the high density of the Finance \& Insurance sector.

As shown in the theoretical framework section, the potential use cases for blockchain technology in financial services make it a crucial industry for further expansion of the fast and tamperproof technology. Even though the competition between different blockchain technology concepts is still ongoing, the key beneficial service offerings provided in this industry receive support from blockchain technology.

A coherent conclusion is possible when evaluating the blockchain landscape from a venture capitalist's perspective. The highest funding is in financial services. It is a result of the already advanced and proven applicability of blockchain technology within the industry. Both views display the potential of blockchain technology within financial services, evaluated by visionary but risky business perspectives.

The second notable industry sector-Information \& Communications-highlights the early stage of blockchain technology. The lack of necessary industry standards for blockchains is embodied in the high density of startups within the Information \& Communications sector. The absolute startup numbers and venture capital funding are indicators as well. This shows that the competition of different technology concepts is still ongoing and results in risky approaches to establish industry-dominating standards with proprietary solutions. Once those standards are established, competition can take place in different applications of the technology. The high density in the Information \& Communication sector is slowing down advancement in further industry applications since it is not clear what kind of blockchain concept-whether 
public/private and permissionless/permissioned-will be the next standard. A comparison between blockchain technology today and the internet in the 1990s may explain the potential further steps for advancement. Once the basic layer standard is in place (i.e., with the ITP or TCP of the internet), competition may move to the upper layer and may have the potential to disrupt existing business models by harnessing the upside potential of the new technology (i.e., speed, cost-efficiency, tamperproof construction).

This assessment of the two largest industry sectors in the sample may explain the results for other industries. Even though the application potential of blockchain technology may be huge, without basic standards the risk of implementing own solutions within other industries is high. Hence, one sees that entrepreneurs are willing to take that risk at the current state of the technology. But venture capitalists are still investing in more advanced industries such as Financial Services and Information \& Communications. In assessing the upside and downside of specific blockchain concepts and further conditions for blockchain applications, further research is necessary to help advance the technology and establish new possibilities for applications implemented in other industries.

Geographically, the blockchain landscape has two emerging clusters in the US and the UK. In terms of investment, however, the US with nearly half of the funds outpaces all other countries in the data sample. The US's trial-and-error culture supports the establishment and growth of startups, and underlines the country's leading position not just for blockchain ventures.

Nevertheless, the high number of uncategorized startups in terms of regional distribution raises an interesting question. With a completely decentralized business model, fully decentralized operating models seem realizable, as seen with the DAO - a decentralized autonomous organization [23]. Henceforth, such applications may result in a global peer-to-peer economy, where national borders do not make a difference [cf. 40]. Therefore, further research needs to lay out the legal, regulatory, and political framework for such a pure global technology.

When evaluating blockchain technology from a diffusion of innovation theoretical perspective, its relative advantage compared to existing technologies together with a vast and high degree of triability (as seen in our empirical results) shows high potential for a broad diffusion of blockchain technology across industries. However, the remaining three attributes, namely compatibility, complexibility, and observability are necessary obstacles for ensure a true disruption of blockchain technology. Further research should elaborate solutions to overcome especially the currently low degrees of compatibility and observability. In conclusion, our analysis shows that the blockchain landscape mirrors the still early stage of the technology but concurrently also identifies its huge potential. The development during the upcoming years will reveal its potential as a merely incremental innovation - or a truly disruptive technology.

\section{References}

[1] A. M. Antonopoulos, Mastering Bitcoin: unlocking digital cryptocurrencies, " O'Reilly Media, Inc.", 2014.

[2] A. Back, Hashcash-a denial of service counter-measure, 2002.

[3] Blockchain Ecosystem Database. Database. 2016. http://www.blockchainangels.eu/startups/browse [Accessed on 15.06.2016]

[4] R. Böhme, N. Christin, B. Edelman and T. Moore, "Bitcoin: Economics, technology, and governance", J. Econ. Perspect. Journal of Economic Perspectives, 29, 2015, pp. 213-238.

[5] E. Bucherer, U. Eisert and O. Gassmann, "Towards Systematic Business Model Innovation: Lessons from Product Innovation Management", Creativity and Innovation Management, 21, 2012, pp. 183-198.

[6] O. Cann, These are the top 10 emerging technologies of 2016, World Economic Forum, 2016.

[7] C. M. Christensen, The innovator's dilemma : when new technologies cause great firms to fail, Harvard Business School Press, Boston, Mass., 1997.

[8] C. M. Christensen, "The Ongoing Process of Building a Theory of Disruption", Journal of Product Innovation Management, 23, 2006, pp. 39-55.

[9] C. M. Christensen, M. E. Raynor and R. McDonald, "What is Disruptive Innovation?", Havard Business Review, 2015, pp. 44-53.

[10] CoinDesk. Database. 2016. http://www.coindesk.com/bitcoin-venture-capital/

[Accessed on 15.06.2016]

[11] Crunchbase. Database. 2016. http://www.crunchbase.com [Accessed on 14.07.2016]

[12] Crunchbase. Database. 2016.

http://www.crunchbase.com [Accessed on 15.06.2016]

[13] Q. Dang, Recommendation for applications using approved hash algorithms, US Department of Commerce, National Institute of Standards and Technology, 2012.

[14] T.-F. Dapp, Are you already experimenting with blockchain? (Fintech \#6), Deutsche Bank Research, 2016.

[15] Deloitte, Blockchain: Democratized trust - Distributed ledgers and the future of value, Tech Trends 2016: Innovation in the digital era, Deloitte University Press, 2016.

[16] W. Diffie and M. Hellman, "New directions in cryptography", IEEE Transactions on Information Theory, 22, 1976, pp. 644-654. 
[17] DTCC, Embracing Disruption - Tapping the Potential of Distributed Ledgers to Improve the Post-Trade Landscape, DTCC, 2016.

[18] European Central Bank, Distributed Ledger Technology, IN FOCUS, Europen Central Bank, 2016, pp. $1-10$.

[19] Federal Information Processing Standards, Secure Hash Standard (SHS), FIPS PUB 180-4, National Institute of Standards and Technology, Gaithersburg, 2015.

[20] R. Garcia and R. Calantone, "A critical look at technological innovation typology and innovativeness terminology: a literature review", Journal of Product Innovation Management, 19, 2002, pp. 110-132.

[21] V. Govindarajan and P. K. Kopalle, "The Usefulness of Measuring Disruptiveness of Innovations Ex Post in Making Ex Ante Predictions", Journal of Product Innovation Management, 23, 2006, pp. 12-18.

[22] T. Hess, C. Matt, A. Benlian and F. Wiesböck, "Options for Formulating a Digital Transformation Strategy", MIS Quarterly Executive, 15, 2016, pp. 123-139.

[23] C. Jentzsch, Decentralized Autonomous Organization to Automate Governance, 2016.

[24] G. O. Karame, E. Androulaki, M. Roeschlin, A. Gervais and S. Čapkun, "Misbehavior in bitcoin: a study of doublespending and accountability", ACM Transactions on Information and System Security (TISSEC), 18, 2015, pp. 2. [25] L. Lamport, M. Pease and R. Shostak, The Byzantine generals problem, SRI International, Menlo Park, Calif, 1982.

[26] M. Mainelli and A. Milne, The Impact and Potential of Blockchain on the Securities Transaction Lifecycle, SWIFT Institute Working Paper, SWIFT Institute, 2016.

[27] C. Markides, "Disruptive innovation: In need of better theory", Journal of Product Innovation Management, 23, 2006, pp. 19-25.

[28] C. Matt, T. Hess and A. Benlian, "Digital Transformation Strategies", Business \& Information Systems Engineering, 57, 2015, pp. 339-343.

[29] J. Mattila, The blockchain phenomenon the disruptive potential of distributed consensus architectures, Berkeley Roundtable on the International Economy (BRIE), University of California, Berkeley, Berkeley, CA, 2016.

[30] McKinsey, Beyond the Hype: Blockchains in Capital Markets, McKinsey Working Papers on Corporate \& Investment Banking, 2015.

[31] McKinsey, Blockchain in insurance - opportunity or threat?, Insurance, McKinsey, 2016.

[32] R. C. Merkle, A Digital Signature Based on a Conventional Encryption Function, in C. Pomerance, ed., Advances in Cryptology - CRYPTO '87: Proceedings, Springer Berlin Heidelberg, Berlin, Heidelberg, 1988, pp. 369-378.

[33] Moody's, Robust, Cost-effective Applications Key to Unlocking Blockchain's Potential Credit Benefits, Moody's Investors Service, Moody's, 2016.

[34] S. Nakamoto, Bitcoin: A Peer-to-Peer Electronic Cash System, 2008.

[35] K. J. O'Dwyer and D. Malone, Bitcoin mining and its energy footprint, Irish Signals \& Systems Conference 2014 and 2014 China-Ireland International Conference on
Information and Communications Technologies (ISSC 2014/CIICT 2014). 25th IET, IET, 2013, pp. 280-285.

[36] A. Osterwalder and Y. Pigneur, Business model generation: a handbook for visionaries, game changers, and challengers, John Wiley \& Sons, 2010.

[37] G. W. Peters and E. Panayi, "Understanding Modern Banking Ledgers through Blockchain Technologies: Future of Transaction Processing and Smart Contracts on the Internet of Money", Available at SSRN 2692487, 2015.

[38] E. M. Rogers, Diffusion of innovations, New York, 2003.

[39] G. M. Schmidt and C. T. Druehl, "When Is a Disruptive Innovation Disruptive?", Journal of Product Innovation Management, 25, 2008, pp. 347-369.

[40] D. Tapscott and A. Tapscott, Blockchain revolution : how the technology behind bitcoin is changing money, business, and the world, Penguin Publishing Group, London, 2016.

[41] G. J. Tellis, "Disruptive Technology or Visionary Leadership?", Journal of Product Innovation Management, 23, 2006, pp. 34-38.

[42] The Economist, The great chain of being sure about things, 2015.

[43] The Economist, The trust machine, 2015.

[44] S. Tongur and M. Engwall, "The business model dilemma of technology shifts", Technovation, 34, 2014, pp. 525-535.

[45] UniCredit, Blockchain Technology and Applications from a Financial Perspective, 2016.

[46] D. Veit, E. Clemons, A. Benlian, P. Buxmann, T. Hess, D. Kundisch, J. M. Leimeister, P. Loos and M. Spann, "Business Models - An Information Systems Research Agenda", Business \& Information Systems Engineering, 6, 2014, pp. 45-53.

[47] VentureRadar. Database. 2016.

https://www.ventureradar.com/ [Accessed on 15.06.2016]

[48] Verbraucherzentrale NRW and

PricewaterhouseCoopers AG, Blockchain - Chance für Energieverbraucher?, 2016.

[49] M. Walport, Distributed Ledger Technology: beyond block chain, UK Government Office for Science, 2016.

[50] WEF, The future of financial infrastructure, World Economic Forum, 2016.

[51] D. Wörner, T. Von Bomhard, Y.-P. Schreier and D. Bilgeri, The Bitcoin Ecosystem: Disruption Beyond Financial Services?, Twenty-Fourth European Conference on Information Systems (ECIS), Istanbul, 2016.

[52] A. Wright and P. De Filippi, Decentralized blockchain technology and the rise of lex cryptographia, 2015.

[53] D. Yu and C. C. Hang, "A Reflective Review of Disruptive Innovation Theory", International Journal of Management Reviews, 12, 2010, pp. 435-452. 